\title{
ON $p$-ADIC DIFFERENTIAL EQUATIONS ON SEMISTABLE VARIETIES II
}

\author{
Valentina Di Proietto* and Atsushi Shiho ${ }^{\dagger}$
}

\begin{abstract}
This paper is a complement to the paper 10. Given an open variety over a DVR with semistable reduction, the author constructed in [10] a fully faithful algebraization functor from the category of certain log overconvergent isocrystals on the special fiber to the category of modules with regular integrable connection on the generic fiber. In this paper, we prove that, with convenable hypothesis, this functor is a tensor functor whose essential image is closed under extensions and subquotients. As a consequence, we can find suitable Tannakian subcategories of $\log$ overconvergent isocrystals and of modules with regular integrable connection on which the algebraization functor is an equivalence of Tannakian categories.
\end{abstract}

\section{Introduction}

Let $V$ be a complete discrete valuation ring of mixed characteristic $(0, p)$, let $K$ be its fraction field and let $k$ be the residue field. Let $X$ be a proper semistable variety over $V$ with special fiber $X_{k}$ and generic fiber $X_{K}$, endowed with a normal crossing divisor $D$. We denote by $U$ the complement of $D$ in $X$, with special fiber $U_{k}$ and generic fiber $U_{K}$. We consider $X$ as a log scheme, with the $\log$ structure associated to $D \cup X_{k}$. We consider $\operatorname{Spec}(V)$ also as a log scheme, with the log structure associated to the closed point.

Let $t$ be the number of irreducible components of $D$. We call a subset $\Sigma$ of the form $\prod_{i=1}^{t} \Sigma_{i}$ in $\mathbb{Z}_{p}^{t}$ (NID) (resp. (NLD)) if, for any $i$ and any $\alpha, \beta \in \Sigma_{i}, \alpha-\beta$ is not a non zero integer (resp. is $p$-adically nonLiouville). For $\Sigma \subset \mathbb{Z}_{p}^{t}$ which is (NID) and (NLD), the author, in [15, introduced the notion of $\Sigma$-unipotent monodromy, as a $p$-adic analogue for isocrystals of the classical notion of regular singularity for modules with integrable connection, in the case where $X$ is smooth. (In [15], we only need the special fibers $X_{k}, U_{k}$. However, this is not our concern here.) In [10 the author generalized this notion to the present situation and constructed a fully faithful algebraization functor from the category of log overconvergent isocrystals on $U_{k}$ with $\Sigma$-unipotent monodromy to the category of modules with integrable connection on $U_{K}$, regular along the generic fiber $D_{K}$ of $D$.

If $\Sigma$ is a subgroup of $\mathbb{Z}_{p}^{t}$, the above algebraization functor would be a tensor functor. However, in this case, $\Sigma$ does not contain any non-zero rational number because of the (NID) hypothesis, and this would not be a natural condition because any log overconvergent isocrystals having geometric origin would not belong to the category.

So we need to consider a more natural condition. As observed in [15, remark 1.4, the notion of $\Sigma$ unipotent monodromy depends only on the reduction $\bar{\Sigma}$ of $\Sigma$ modulo $\mathbb{Z}^{t}$. So we can call the notion of $\Sigma$-unipotent monodromy as $\bar{\Sigma}$-unipotent monodromy. Then we can start from a subset $\bar{\Sigma}=\prod_{i=1}^{t} \bar{\Sigma}_{i}$ of $\left(\mathbb{Z}_{p} / \mathbb{Z}\right)^{t}$ satisfying the (NLD) condition (defined in a way similar to above) and we can consider the category of log overconvergent isocrystals with $\bar{\Sigma}$-unipotent monodromy. If moreover $\bar{\Sigma}$ is a subgroup of $\left(\mathbb{Z}_{p} / \mathbb{Z}\right)^{t}$, this category is a rigid abelian tensor category. Typical example of $\bar{\Sigma}$ is the group $\left(\mathbb{Z}_{(p)} / \mathbb{Z}\right)^{t}$, in which case any $\log$ overconvergent isocrystals having geometric origin would belong to the category.

To construct the algebraization functor in this setting, we choose a section $\tau: \mathbb{Z}_{p} / \mathbb{Z} \rightarrow \mathbb{Z}_{p}$ of the canonical projection and we proceed as in [10]: It is obtained as the composite of the following four functors.

\footnotetext{
*Institut de Recherche Mathématique Avancée, Université de Strasbourg, 7 rue René Descartes, Strasbourg 67084, France.

${ }^{\dagger}$ Graduate School of Mathematical Sciences, the University of Tokyo, 3-8-1 Komaba, Meguro-ku, Tokyo 153-8914, Japan. Mathematics Subject Classification (2010): 12H25, 14F35.
} 
(1) The equivalence of categories between the category of log overconvergent isocrystals on $U_{k}$ with $\bar{\Sigma}$ unipotent monodromy $\left(=\tau(\bar{\Sigma}):=\prod_{i=1}^{t} \tau\left(\Sigma_{i}\right)\right.$-unipotent monodromy) and the category of log convergent isocrystals on $X_{k}$ with exponents in $\tau(\bar{\Sigma})$, which is the $p$-adic semistable version of the canonical logarithmic extension of Deligne, André-Baldassarri.

(2) The fully faithful functor of 'forgetting the convergence condition' from the category of log convergent isocrystals on $X_{k}$ with exponents in $\tau(\bar{\Sigma})$ to the category of modules with integrable log connection on the $p$-adic completion $\hat{X}$ of $X$ with exponents in $\tau(\bar{\Sigma})$.

(3) The GAGA equivalence between the category of modules with integrable log connection on $\hat{X}$ with exponents in $\tau(\bar{\Sigma})$ and that on $X_{K}$ with exponents in $\tau(\bar{\Sigma})$.

(4) The fully faithful restriction functor from the category of modules with integrable log connection on $X_{K}$ with exponents in $\tau(\bar{\Sigma})$ to that of modules with regular integrable connection on $U_{K}$, whose essential image lands in the subcategory of objects with exponents in $\bar{\Sigma}$.

Note that, since $\tau(\bar{\Sigma})$ is not necessarily a group, the functor (1) is not necessarily a tensor functor and the composite, which we denote by $\psi_{\tau}$, depends a priori on the choice of $\tau$. Nevertheless, we prove in this paper that the functor $\psi_{\tau}$ is a tensor functor which is independent of $\tau$. We prove this by looking carefully the definition of $\psi_{\tau}$ and using techniques developed in [15], [16. We also prove that the essential image of the functor $\psi_{\tau}$ is closed by extensions and subquotients.

As a consequence, we have the surjection of Tannaka duals when $U_{K}$ is connected and admits a $K$ rational point. Also, we can find Tannakian subcategories of $\log$ overconvergent isocrystals on $U_{k}$ with $\bar{\Sigma}$-unipotent monodromy and of modules with regular integrable connection on $U_{K}$ strictly containing the unipotent parts, on which the functor $\psi_{\tau}$ becomes an equivalence of categories. (It suffices to consider the smallest subcategory containing a $\log$ overconvergent isocrystal $\mathcal{E}$ with $\bar{\Sigma}$-unipotent monodromy and closed under extensions, subquotients, tensors and duals.)

The content of each section is as follows. In the second section, we give preliminaries and fix some notations on our geometric setting. In the third section, we recall the definitions and the results in [10] concerning log- $\nabla$-modules on rigid analytic spaces and isocrystals. In the fourth section, we recall the definitions and the results in [10, [1] concerning modules with integrable connection on (formal log) schemes. In the fifth section, we give a proof of our results explained above.

\section{Preliminaries and notation}

Let $V$ be a complete discrete valuation ring of mixed characteristic $(0, p)$ with uniformizer $\pi$, let $K$ be its fraction field and let $k$ be the residue field. Let $X$ be a proper semistable variety over $\operatorname{Spec}(V)$ with special fiber $X_{k}$ and generic fiber $X_{K}$, and let $D$ be a horizontal normal crossing divisor. This means that étale locally we have a cartesian diagram

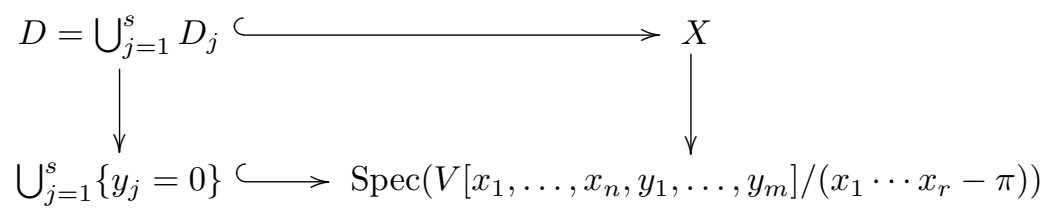

where the vertical maps are étale and the horizontal maps are closed immersions.

We consider on $X$ the $\log$ structure $M$ associated to the divisor with normal crossings $X_{k} \cup D$ in $X$; the structural morphism from $X$ to $\operatorname{Spec}(V)$ extends to a log smooth morphism of log schemes $(X, M) \rightarrow$ $(\operatorname{Spec}(V), N)$, where $\operatorname{Spec}(V)$ is endowed with the $\log$ structure $N$ associated to the closed point. We denote the pull-back of the $\log$ structure $M$ (resp. $N$ ) to $X_{k}$ or $X_{K}(\operatorname{resp}$. $\operatorname{Spec}(k)$ or $\operatorname{Spec}(K)$ ) also by $M$ (resp. $N$ ), by abuse of notation. Note that the $\log$ structure $N$ on $\operatorname{Spec}(K)$ is the trivial $\log$ structure. Let us put 
$U:=X \backslash D$ and denote the canonical open immersion $U \hookrightarrow X$ by $j$. We denote the pull-back of the $\log$ structure $M$ to $U$ again by $M$.

We denote by $\hat{X}$ and $\hat{D}$ the $p$-adic completion of $X$ and $D$, respectively. We denote the pull-back of the $\log$ structure $M$ to $\hat{X}$ again by $M$ and the pull-back of the $\log$ structure $N$ to $\operatorname{Spf}(V)$ again by $N$. Étale locally we have a cartesian diagram of formal schemes

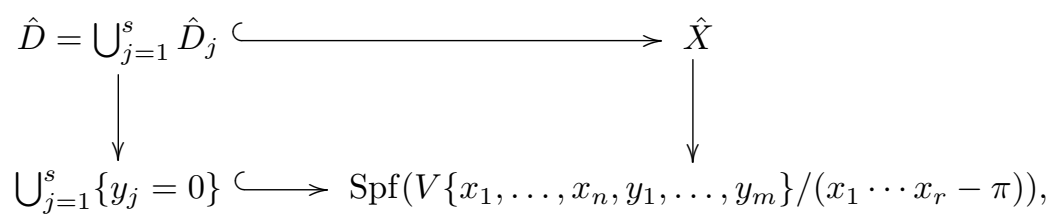

where the vertical maps are étale and the horizontal maps are closed immersions.

Let $\hat{X}_{\text {sing }}$ and $\hat{D}_{\text {sing }}$ be the singular loci of $\hat{X}$ and $\hat{D}$ respectively, and we define $\hat{X}^{\circ}, \hat{D}^{\circ}$ as follows:

$$
\hat{X}^{\circ}=\hat{X} \backslash\left(\hat{X}_{\text {sing }} \cup \hat{D}_{\text {sing }}\right), \quad \hat{D}^{\circ}=\hat{D} \backslash\left(\hat{X}_{\text {sing }} \cup \hat{D}_{\text {sing }}\right)=\hat{X}^{\circ} \cap \hat{D} .
$$

When we consider the situation étale locally and fix a diagram (2), we can define the open formal subscheme $\hat{X}_{i}^{\circ}$ of $\hat{X}$ by $\hat{X}_{i}^{\circ}:=\left\{x_{i^{\prime}} \neq 0\right.$ for $\left.\forall i^{\prime} \neq i\right\}$ and the open formal subscheme $\hat{X}_{i, j}^{\circ}$ of $\hat{X}_{i}^{\circ}$ by $\hat{X}_{i, j}^{\circ}:=\left\{x_{i^{\prime}} \neq\right.$ 0 for $\forall i^{\prime} \neq i, y_{j^{\prime}} \neq 0$ for $\left.\forall j^{\prime} \neq j\right\}$. Moreover we define $\hat{D}_{i, j}^{\circ}$ by $\hat{D}_{i, j}^{\circ}:=\hat{X}_{i, j}^{\circ} \cap \hat{D}=\hat{X}_{i, j}^{\circ} \cap \hat{D}_{j}$. With this notations we have the following relations:

$$
\coprod_{i} \hat{X}_{i}^{\circ}=\hat{X}-\hat{X}_{\text {sing }}, \quad \coprod_{i, j} \hat{X}_{i, j}^{\circ}=\hat{X}^{\circ}, \quad \coprod_{i, j} \hat{D}_{i, j}^{\circ}=\hat{D}^{\circ} .
$$

If we denote by the subscript ${ }_{K}$ the rigid analytic space associated to a formal scheme, then the sets $\hat{D}_{i, j ; K}^{\circ}$ and $\hat{X}_{i, j ; K}^{\circ}$ can be described as follows:

$$
\begin{gathered}
\hat{X}_{i, j ; K}^{\circ}=\left\{P \in \hat{X}_{K}\left|\forall i^{\prime} \neq i\right| x_{i^{\prime}}(P)\left|=1, \forall j^{\prime} \neq j\right| y_{j^{\prime}}(P) \mid=1\right\}, \\
\hat{D}_{i, j ; K}^{\circ}=\left\{P \in \hat{X}_{K}\left|\forall i^{\prime} \neq i\right| x_{i^{\prime}}(P)\left|=1, \forall j^{\prime} \neq j\right| y_{j^{\prime}}(P) \mid=1, y_{j}(P)=0\right\} .
\end{gathered}
$$

Finally we denote by $\hat{U}$ the open formal subscheme complement of $\hat{D}$ in $\hat{X}$.

\section{$3 \quad$ Log- $\nabla$-modules and isocrystals}

We recall the notion of $(\log -) \nabla$-modules on rigid spaces defined and used by Kedlaya in [1] and by the author in [15], [16], 17] and [14.

Definition 3.1. Let $Y$ be a smooth rigid analytic space over $K . A \nabla$-module on $Y / K$ is a coherent $\mathcal{O}_{Y^{-}}$ module $E$ equipped with an integrable connection

$$
\nabla: E \rightarrow E \otimes \Omega_{Y / K}^{1}
$$

with $\Omega_{Y / K}^{1}$ the sheaf of continuous one forms on the rigid analytic space $Y$.

It is known that, for a $\nabla$-module $(E, \nabla), E$ is automatically a locally free $\mathcal{O}_{Y}$-module of finite rank.

Definition 3.2. Let $Y$ be a smooth rigid analytic space over $K$, with $y_{1}, \ldots, y_{n} \in \Gamma\left(Y, \mathcal{O}_{Y}\right)$. A log- $\nabla$-module on $Y / K$ with respect to $y_{1}, \ldots, y_{n}$ is a locally free $\mathcal{O}_{Y}$-module $E$ of finite rank equipped with an integrable connection

$$
\nabla: E \rightarrow E \otimes \omega_{Y / K}^{1}
$$

where

$$
\omega_{Y / K}^{1}=\left(\Omega_{Y / K}^{1} \oplus \bigoplus_{j=1}^{n} \mathcal{O}_{Y} \mathrm{~d} \log y_{j}\right) / L
$$

with $L$ the coherent sub $\mathcal{O}_{Y}$-module generated by $\left(\mathrm{d} y_{j}, 0\right)-\left(0, y_{j} \mathrm{~d} \log y_{j}\right)$ for $1 \leq j \leq n$. 
Contrary to the case of $\nabla$-modules, the local freeness should be put in the definition in the case of $\log -\nabla$-modules.

Remark 3.3. When $y_{1}, \ldots, y_{n}$ are invertible, the notion of a $\log -\nabla$-module on $Y / K$ with respect to $y_{1}, \ldots, y_{n}$ is the same as the notion of a $\nabla$-module on $Y / K$.

For $j \in\{1, \ldots, n\}$ there is a natural map

$$
\Omega_{Y / K}^{1} \oplus \bigoplus_{j^{\prime} \neq j} \mathcal{O}_{Y} \operatorname{d} \log y_{j} \rightarrow \omega_{Y / K}^{1},
$$

whose image we denote by $M_{j}$. If the zero loci $D_{j}$ of $y_{j}(1 \leq j \leq n)$ in $Y$ are smooth and meet transversally, we have the isomorphisms $\omega_{Y / K}^{1} / M_{j} \cong \mathcal{O}_{D_{j}} \mathrm{~d} \log y_{j} \cong \mathcal{O}_{D_{j}}$.

Definition 3.4. Let $Y, y_{1}, \ldots, y_{n}$ be as above and assume that the zero loci $D_{j}$ of $y_{j}(1 \leq j \leq n)$ in $Y$ are affinoid, smooth and meet transversally. For a $\log$ - $\nabla$-module $(E, \nabla)$ on $Y$, let res $j$ be the endomorphism on $E \otimes \mathcal{O}_{D_{j}}$ induced by

$$
E \rightarrow E \otimes \omega_{Y / K}^{1} \rightarrow E \otimes \omega_{Y / K}^{1} / M_{j} \cong E \otimes \mathcal{O}_{D_{j}} \mathrm{~d} \log y_{j} \cong E \otimes \mathcal{O}_{D_{j}} .
$$

We call it the residue of $E$ along $D_{j}$. Thanks to proposition-definition 1.24 in [17] (based on proposition 1.5.3 of [3] ), we know that there exists a minimal monic polynomial $P_{j} \in K[T]$ such that $P_{j}\left(\operatorname{res}_{j}\right)=0$. We call the roots of $P_{j}$ the exponents of $E$ along $D_{j}$.

Remark 3.5. It is easy to see that the above definition works also in the case where $Y$ has a finite admissible covering $Y=\bigcup_{i=1}^{m} Y_{i}$ such that each $Y_{i} \cap D_{j}$ are affinoids: Indeed, we can define the minimal monic polynomial in the definition for each $Y_{i} \cap D_{j}$, and it suffices to define the polynomial $P_{j}$ as the least common multiple of them.

For an aligned interval (an interval $I$ such that any endpoint is contained in $\Gamma^{*}:=\sqrt{\left|K^{*}\right|} \cup\{0\}$ ), we define an $n$-dimensional rigid analytic polyannulus $A_{K}^{n}(I)$ over $K$ with radius in $I$ as

$$
A_{K}^{n}(I)=\left\{\left(t_{1}, \ldots, t_{n}\right) \in \mathbb{A}_{K}^{n, r i g} \| t_{j} \mid \in I \forall j=1, \ldots, n\right\} .
$$

If $\Sigma=\prod_{j=1}^{n} \Sigma_{j}$ is a subset of $\mathbb{Z}_{p}^{n}$ and $\xi:=\left(\xi_{1}, \ldots, \xi_{n}\right)$ an element of $\Sigma$, we denote by $M_{\xi}$ the log- $\nabla$-module on $A_{K}^{n}(I)$ defined by $\left(\mathcal{O}_{A_{K}^{n}(I)}, d+\sum_{j=1}^{n} \xi_{i} \operatorname{dlog} t_{j}\right)$.

Definition 3.6. Let $Y$ be a smooth rigid analytic space, $I$ an aligned interval and $\Sigma=\prod_{j=1}^{n} \Sigma_{j} \subset \mathbb{Z}_{p}^{n}$. We regard that the smooth rigid analytic space $Y \times A_{K}^{n}(I)$ is endowed with the sections $t_{1}, \ldots, t_{n}$, which are the coordinates of the polyannulus $A_{K}^{n}(I)$. We say that a $\log$ - $\nabla$-module $E$ on $Y \times A_{K}^{n}(I)$ is $\Sigma$-unipotent if there exists a filtration

$$
0 \subset E_{1} \subset \cdots \subset E_{t}=E
$$

by subobjects such that every successive quotient $E_{i} / E_{i-1}$ has the form $\pi_{1}^{*} F \otimes \pi_{2}^{*}\left(M_{\xi}\right)$, where $\pi_{1}: Y \times A_{K}^{n}(I) \rightarrow$ $Y$ denotes the first projection, $\pi_{2}: Y \times A_{K}^{n}(I) \rightarrow A_{K}^{n}(I)$ the second, $F$ is a $\nabla$-module on $Y$ and $M_{\xi}$ the $\log -\nabla$-module we defined before with $\xi \in \Sigma$.

Remark 3.7. As observed in [15] 1.4, if I does not contain 0, the notion of $\Sigma$-unipotence depends only on the image $\bar{\Sigma}$ of $\Sigma$ in $\left(\mathbb{Z}_{p} / \mathbb{Z}\right)^{n}$ in the following sense: any log- $\nabla$-module $(E, \nabla)$ in $Y \times A_{K}^{n}(I)$ is $\Sigma$-unipotent if and only if for any section $\tau=\prod_{j=1}^{n} \tau_{j}:\left(\mathbb{Z}_{p} / \mathbb{Z}\right)^{n} \rightarrow\left(\mathbb{Z}_{p}\right)^{n}$ of the canonical projection is $\tau(\bar{\Sigma})$-unipotent. So, in the following, we often call the $\Sigma$-unipotence as the $\bar{\Sigma}$-unipotence.

As in definition 1.8 of [15] we can give following definition. 
Definition 3.8. A set $\Sigma \subset \mathbb{Z}_{p}$ is called (NID) (resp. (NLD)) if for any $\alpha, \beta \in \Sigma, \alpha-\beta$ is not a non zero integer (resp. is p-adically non-Liouville). A set $\Sigma=\prod_{j=1}^{n} \Sigma_{j} \subset \mathbb{Z}_{p}^{n}$ is called (NID) (resp. (NLD)) if $\Sigma_{j}$ is (NID) (resp. (NLD)) for any $j=1, \ldots, n$.

$A$ set $\Sigma \subset \mathbb{Z}_{p} / \mathbb{Z}$ is called (NLD) if, for any section $\tau: \mathbb{Z}_{p} / \mathbb{Z} \rightarrow \mathbb{Z}_{p}$ of the projection and for any $\alpha, \beta$ $\in \Sigma, \alpha-\beta$ is p-adically non-Liouville. A set $\Sigma=\prod_{j=1}^{n} \Sigma_{j} \subset\left(\mathbb{Z}_{p} / \mathbb{Z}\right)^{n}$ is (NLD) if $\Sigma_{j}$ is (NLD) for any $j=1, \ldots, n$.

Now we recall the theorem of logarithmic extension for log overconvergent isocrystals in semistable situation proven in theorem 5, section 12 of [10], which generalizes of the main theorem of [15]. We start recalling the notion of log overconvergent isocrystal with $\Sigma$-unipotent monodromy, and the notion of $\log$ convergent isocrystal with exponents in $\Sigma$.

We consider $I^{\dagger}\left(U_{k} / V\right)^{\log }$, the category of overconvergent log isocrystals on the log pair $\left(\left(U_{k}, M\right),\left(X_{k}, M\right)\right)$ over $(\operatorname{Spf}(V), N)$, as defined in section 4 in [13]. (See section 10 in [10] for the definition in the present situation. Note that we use simpler notation in this paper to lighten the notation.) In the present situation, it is defined as a coherent $j^{\dagger} \mathcal{O}_{\hat{X}_{K}}$-module endowed with a stratification on the log tubular neighborhood

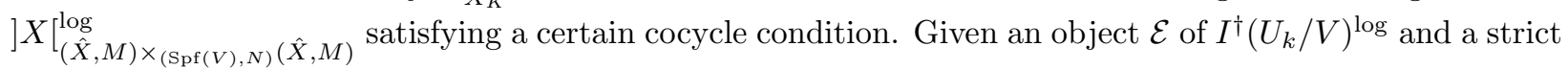
neighborhood $W$ of $] U_{k}[\hat{X}$ in $] X_{k}\left[_{\hat{X}}=\hat{X}_{K}\right.$ which is small enough, $\mathcal{E}$ induces a $\nabla$-module $(E, \nabla)$ on $W$. (This follows from the fact that the $\log$ structure $M$ on $\hat{U}$ comes only from the special fiber $U_{k}$ of $U$, not from $\hat{D}$.) So $E$ is locally free, and this implies that $\mathcal{E}$ is automatically a locally free $j^{\dagger} \mathcal{O}_{\hat{X}_{K}}$-module of finite rank.

If étale locally we are in the situation described in $22, W$ contains $\hat{D}_{i, j ; K}^{\circ} \times A_{K}^{1}([\lambda, 1))$ for all $i, j$ and for some $\lambda \in(0,1) \cap \Gamma^{*}$ and so we can define the restriction of $(E, \nabla)$ on it, which is a log- $\nabla$-module on $\hat{D}_{i, j ; K}^{\circ} \times A_{K}^{1}([\lambda, 1))$ (for the reason that we can put the term 'log-' here, see remark 3.3.

Let $\bar{\Sigma}=\prod_{h=1}^{t} \bar{\Sigma}_{h}$ be a subset of $\left(\mathbb{Z}_{p} / \mathbb{Z}\right)^{t}$ which is (NLD), where $t$ is the number of irreducible components of $D=\bigcup_{h=1}^{t} D^{h}$. If there exists a small enough étale covering $\coprod_{l} \phi_{l}: \coprod_{l} \hat{X}_{l} \rightarrow \hat{X}$ such that every $\hat{X}_{l}$ has a diagram

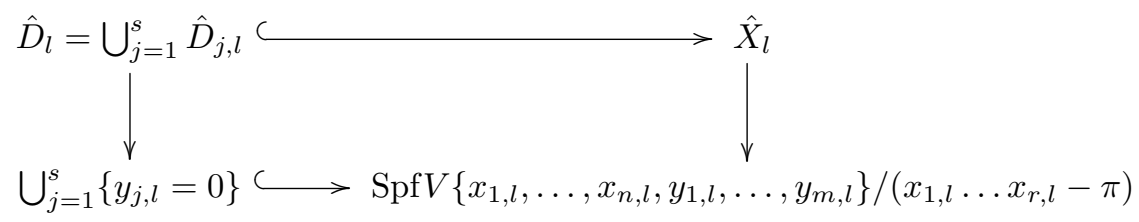

as in (2), then we can define a function of sets $h:\{1, \ldots, s\} \rightarrow\{1, \ldots, t\}$ for each $l$ as follows: for any $1 \leq j \leq s, \phi_{l}\left(\hat{D}_{j, l}\right)$ is contained in one irreducible component $D^{h(j)}$ of $D$.

Definition 3.9. A log overconvergent isocrystal $\mathcal{E} \in I^{\dagger}\left(U_{k} / V\right)^{\log }$ has $\bar{\Sigma}$-unipotent monodromy if there exists an étale covering $\coprod_{l} \phi_{l}: \coprod_{l} \hat{X}_{l} \rightarrow \hat{X}$ such that every $\hat{X}_{l}$ has a diagram (3) with $\hat{D}_{l}=\phi_{l}^{-1}(\hat{D})$ and the restriction of the $\nabla$-module associated to $\mathcal{E}$ to $\hat{D}_{i, j, l ; K}^{\circ} \times A_{K}^{1}([\lambda, 1))$ is $\bar{\Sigma}_{h(j)}$-unipotent for all $l, i$ and $j$. We denote by $I^{\dagger}\left(U_{k} / V\right)^{\log , \bar{\Sigma}}$ the category of log overconvergent isocrystals with $\bar{\Sigma}$-unipotent monodromy .

Remark 3.10. Here we used a slightly different formulation from [10]: $t$ there was the number of irreducible

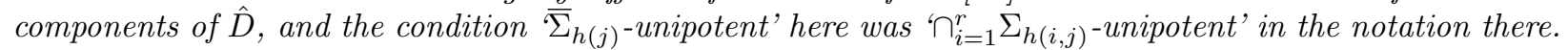
However, this does not make any serious difference.

The notion of $\bar{\Sigma}$-unipotent monodromy is independent of the choice of the étale covering and the diagram (3) which are chosen in definition 3.9 (proposition 17 of section 12 of [10]).

We also consider the category of locally free log convergent isocrystals as defined in [12] section 5. (See sections 4 and 8 in [10] for the discussion in the present situation.) It is the category of locally free isocrystals on the $\log$ convergent site $\left(\left(X_{k}, M\right) /(\operatorname{Spf}(V), N)\right)_{\text {conv }}$; we denote it by $I_{\text {conv }}\left(X_{k} / V\right)^{\log }$. In the local situation as in $(2), \mathcal{E} \in I_{\text {conv }}\left(X_{k} / V\right)^{\log }$ induces a $\log -\nabla$-module on $\hat{X}_{K}$ with respect to $y_{1}, \ldots, y_{s}$.

Let $\Sigma=\prod_{h=1}^{t} \bar{\Sigma}_{h}$ be a subset of $\left(\mathbb{Z}_{p} / \mathbb{Z}\right)^{t}$, where $t$ is the number of the irreducible components of $D=\bigcup_{h=1}^{t} D^{h}$ and let $\tau: \mathbb{Z}_{p} / \mathbb{Z} \rightarrow \mathbb{Z}_{p}$ be a section of the canonical surjection $\mathbb{Z}_{p} \rightarrow \mathbb{Z}_{p} / \mathbb{Z}$. 
Definition 3.11. A locally free convergent isocrystal $\mathcal{E}$ has exponents in $\tau(\bar{\Sigma}):=\prod_{h=1}^{t} \tau\left(\bar{\Sigma}_{h}\right)$ if there exists an étale covering $\coprod_{l} \phi_{l}: \coprod_{l} \hat{X}_{l} \rightarrow \hat{X}$ such that every $\hat{X}_{l}$ has a diagram (3) with $\hat{D}_{l}:=\phi_{l}^{-1}(\hat{D})$ and the $\log -\nabla$-module $E_{\mathcal{E}, l}$ on $\hat{X}_{l ; K}$ induced by $\mathcal{E}$ has exponents in $\tau\left(\bar{\Sigma}_{h(j)}\right)$ along $\hat{D}_{j, l ; K}$ for all $l$ and $j$. We denote by $I_{\text {conv }}\left(X_{k} / V\right)^{\log , \tau(\bar{\Sigma})}$ the category of locally free log convergent isocrystals with exponents in $\tau(\bar{\Sigma})$.

The notion of exponents in $\tau(\bar{\Sigma})$ is independent of the choice of the étale covering and the diagram (3), which are chosen in definition 3.11 (lemma 2 of section 8 of [10]).

Definition 3.12. Let $\bar{\Sigma}=\prod_{h=1}^{t} \bar{\Sigma}_{h}$ be a subset of $\left(\mathbb{Z}_{p} / \mathbb{Z}\right)^{t}$. We say that $\bar{\Sigma}$ is (NLG) if it is a subgroup of $\left(\mathbb{Z}_{p} / \mathbb{Z}\right)^{t}$ and if for any section $\tau: \mathbb{Z}_{p} / \mathbb{Z} \rightarrow \mathbb{Z}_{p}$ the set $\tau(\bar{\Sigma}):=\prod_{h=1}^{t} \tau\left(\bar{\Sigma}_{h}\right)$ consists of elements that are, component by component, $p$-adic non-Liouville numbers.

Let us remark that if $\bar{\Sigma}$ is (NLG) and if $\tau: \mathbb{Z}_{p} / \mathbb{Z} \rightarrow \mathbb{Z}_{p}$ is a section, $\tau(\bar{\Sigma})$ is (NID) and (NLD). Hence, as in remark 3.17 of [15], theorem 5 of section 12 of [10] implies the following theorem:

Theorem 3.13. We fix a set $\bar{\Sigma}=\prod_{h=1}^{t} \bar{\Sigma}_{h} \in\left(\mathbb{Z}_{p} / \mathbb{Z}\right)^{t}$, where $t$ is the number of the irreducible components of $\hat{D}=\bigcup_{h=1}^{t} \hat{D}^{t}$ in $\hat{X}$ and we require that $\bar{\Sigma}$ is $(N L G)$. Then, for each section $\tau: \mathbb{Z}_{p} / \mathbb{Z} \rightarrow \mathbb{Z}_{p}$, the restriction functor

$$
j_{\tau}^{\dagger}: I_{\text {conv }}\left(X_{k} / V\right)^{\log , \tau(\bar{\Sigma})} \longrightarrow I^{\dagger}\left(U_{k} / V\right)^{\log , \bar{\Sigma}},
$$

which is induced by the tensor functor

$$
j^{\dagger}: I_{\text {conv }}\left(X_{k} / V\right)^{\log } \longrightarrow I^{\dagger}\left(U_{k} / V\right)^{\log },
$$

is a well-defined functor. It is indeed an equivalence of categories.

Definition 3.14. Let the hypotheses be as in theorem 3.13 and let $\mathcal{E} \in I^{\dagger}\left(U_{k} / V\right)^{\log , \bar{\Sigma}}$. We define $\bar{\Sigma}_{\mathcal{E}} \subset \bar{\Sigma}$ as the minimum subset of the form $\bar{\Sigma}_{\mathcal{E}}=\prod_{h=1}^{t} \bar{\Sigma}_{\mathcal{E}, h}$ such that $\mathcal{E}$ has $\bar{\Sigma}_{\mathcal{E}}$-unipotent monodromy.

Remark 3.15. For a given log overconvergent isocrystal $\mathcal{E}$ the set $\bar{\Sigma}_{\mathcal{E}}$ is a finite set. Indeed, we can suppose that the étale covering $\coprod_{l} \phi_{l}: \coprod_{l} \hat{X}_{l} \rightarrow \hat{X}$ of definition 3.9 is finite, hence we have a finite number of annuli $\hat{D}_{i, j, l ; K}^{\circ} \times A_{K}^{1}([\lambda, 1))$, and a finite number of $M_{\xi}$ 's appears in the expression in definition 3.6 for the $\log$ - $\nabla$-module on each $\hat{D}_{i, j, l ; K}^{\circ} \times A_{K}^{1}([\lambda, 1))$ induced by $\mathcal{E}$.

Definition 3.16. Let $\Sigma_{1}:=\prod_{j=1}^{n} \Sigma_{1, j}$ and $\Sigma_{2}:=\prod_{j=1}^{n} \Sigma_{2, j}$ be subsets of $\mathbb{Z}_{p}^{n}$; we denote the set

$$
\prod_{j=1}^{n}\left\{\xi_{1, j} \pm \xi_{2, j} \mid \xi_{1, j} \in \Sigma_{1, j}, \xi_{2, j} \in \Sigma_{2, j}\right\}
$$

by $\Sigma_{1} \pm \Sigma_{2}$. In particular for $\boldsymbol{\alpha}=\left(\alpha_{1}, \ldots, \alpha_{n}\right) \in \mathbb{Z}_{p}^{n}$, we put

$$
\Sigma \pm \boldsymbol{\alpha}:=\prod_{j=1}^{n}\left\{\xi_{j} \pm \alpha_{j} \mid \xi_{j} \in \Sigma_{j}\right\}
$$

We see that $I^{\dagger}\left(U_{k} / V\right)^{\log , \bar{\Sigma}}$ is a rigid abelian tensor category under convenable hypothesis.

Proposition 3.17. If $\bar{\Sigma}$ is $(N L G)$, the category $I^{\dagger}\left(U_{k} / V\right)^{\log , \bar{\Sigma}}$ is a rigid abelian tensor category.

Proof. First, the category $I^{\dagger}\left(U_{k} / V\right)^{\log }$ is an abelian tensor category having internal hom's; this can be seen easily from definition (of an object as a coherent module endowed with stratification). Moreover, using the fact that the objects are locally free, one can see that it is rigid.

Hence it suffices to prove that the category $I^{\dagger}\left(U_{k} / V\right)^{\log , \bar{\Sigma}}$ is closed by the formation of subquotient, tensor product and dual in $I^{\dagger}\left(U_{k} / V\right)^{\log }$. 
Since the definition of $\bar{\Sigma}$-unipotent monodromy is given étale locally, it is sufficient to check these properties for the $\bar{\Sigma}$-unipotence of $\log -\nabla$-modules on $\hat{D}_{i, j, l ; K}^{\circ} \times A_{K}^{1}([\lambda, 1))$ with the same notation as in definition 3.9 .

The notion of $\bar{\Sigma}$-unipotence is closed by the formation of subquotients by proposition 1.17 of [15. So we need to prove that the notion of $\bar{\Sigma}$-unipotence is closed by the formation of tensor product and dual. One can check it easily by using the isomorphisms $M_{\xi} \otimes M_{\xi^{\prime}}=M_{\xi+\xi^{\prime}}, M_{\xi}^{*}=M_{-\xi}$ and the assumption that $\bar{\Sigma}$ is a subgroup of $\left(\mathbb{Z}_{p} / \mathbb{Z}\right)^{t}$.

\section{Formal and algebraic modules with integrable connection}

In this section, we recall the definition of the category of modules with integrable connection on (formal $\log$ ) schemes. First we recall the definition in the case of formal $(\log )$ schemes.

Definition 4.1. We define the category $\widehat{M I C}(\hat{U} / V)$ of modules with integrable connection on $\hat{U}$ over $V$ as follows: An object is an isocoherent sheaf $E$ (a sheaf of the form $K \otimes_{V} \tilde{E}$, where $\tilde{E}$ is a coherent $\mathcal{O}_{\hat{U}}$-module) endowed with an integrable connection

$$
\nabla: E \rightarrow E \otimes \Omega_{\hat{U} / V}^{1}
$$

where $\Omega_{\hat{U} / V}^{1}$ is the sheaf of continuous one forms on $\hat{U}$ over $V$. For two objects $\left(E, \nabla_{E}\right)$ and $\left(F, \nabla_{F}\right)$ in $\widehat{M I C}(\hat{U} / V)$, a morphism $f:\left(E, \nabla_{E}\right) \rightarrow\left(F, \nabla_{F}\right)$ is defined to be a $K \otimes_{V} \mathcal{O}_{\hat{U}}$-linear homomorphism $f: E \rightarrow F$ such that $f \circ \nabla_{E}=\nabla_{F} \circ f$. It is known that $E$ is automatically 'locally free' (a projective $K \otimes_{V} \mathcal{O}_{\hat{U}}$-module locally in Zariski topology).

Definition 4.2. We define the category $\widehat{M I C}(\hat{X} / V)^{\log }$ of modules with integrable connection on $(\hat{X}, M)$ over $(\operatorname{Spf}(V), N)$ as follows: An object in $\widehat{M I C}(\hat{X} / V)^{\log }$ is a locally free (in the sense of the above definition) isocoherent sheaf $E$ endowed with an integrable connection

$$
\nabla: E \rightarrow E \otimes \omega_{(\hat{X}, M) /(V, N)}^{1}
$$

where $\omega_{(\hat{X}, M) /(V, N)}^{1}$ is the sheaf of continuous one forms of $(X, M)$ over $(\operatorname{Spf}(V), N)$ in the framework of fine log formal schemes.

Given $(E, \nabla) \in \widehat{M I C}(\hat{X} / V)^{\log }$ and given a diagram (2), we can define the exponents of $(E, \nabla)$ along each $\hat{D}_{j}$ : the definition is perfectly analogous to the one given for $\log -\nabla$-modules on rigid analytic spaces (definition 3.4. We can globalize the definition of exponents as in definition 3.11 in the following way.

Definition 4.3. We fix a set $\Sigma=\prod_{h=1}^{t} \Sigma_{h} \subset \bar{K}^{t}$, where $t$ is the number of irreducible components of $D=\cup_{h=1}^{t} D^{h}$. We say that an object $(E, \nabla)$ in $\widehat{M I C}(\hat{X} / V)^{\log }$ has exponents in $\Sigma$ if there exists an étale covering $\coprod_{l} \phi_{l}: \coprod_{l} \hat{X}_{l} \rightarrow \hat{X}$ such that every $\hat{X}_{l}$ has a diagram $(3)$ with $\hat{D}_{l}=\phi_{l}^{-1}(\hat{D})$ and for every $l$ and $j$, the restriction of $(E, \nabla)$ to $\hat{X}_{l}$ has exponents in $\Sigma_{h(j)}$ along $\hat{D}_{j, l}$, where $\phi_{l}\left(\hat{D}_{j, l}\right) \subset D^{h(j)}$. We denote by $\widehat{M I C}(\hat{X} / V)^{\log , \Sigma}$ the category of modules with integrable connection on $(\hat{X}, M)$ over $(\operatorname{Spf}(V), N)$ with exponents in $\Sigma$.

One can prove by arguing as in the analytic case ([10] lemma 2 of section 8$)$ that the above definition does not depend on the choice of the étale covering and the diagram (3).

Remark 4.4. If we are given a diagram 20, the category $\widehat{M I C}(\hat{X} / V)^{\log }$ is naturally equivalent to the category of log- $\nabla$-modules on $\hat{X}_{K}$ with respect to $y_{1}, \ldots, y_{s}$. Under this equivalence, the notions of 'exponents in $\Sigma$ ' coincide.

Next we recall the definition in the case of usual (log) schemes. 
Definition 4.5. We define the category $\operatorname{MIC}\left(U_{K} / K\right)$ of modules with integrable connection on the scheme $U_{K}$ as follows: An object in $M I C\left(U_{K} / K\right)$ is a coherent $\mathcal{O}_{U_{K}}$-module $E$ endowed with an integrable connection

$$
\nabla: E \rightarrow E \otimes \Omega_{U_{K} / K}^{1}
$$

It is known that $E$ is automatically locally free.

Definition 4.6. We define the category $M I C\left(X_{K} / K\right)^{\log }$ of modules with integrable connection on $\left(X_{K}, M\right)$ over $K$ as follows: An object in $M I C\left(X_{K} / K\right)^{\log }$ is a locally free $\mathcal{O}_{X_{K}}$-module $E$ of finite rank endowed with an integrable connection

$$
\nabla: E \rightarrow E \otimes \omega_{\left(X_{K}, M\right) / K}^{1}
$$

where $\omega_{\left(X_{K}, M\right) / K}^{1}$ is the sheaf of logarithmic one forms on $\left(X_{K}, M\right)$ over $K$ in the framework of fine log schemes.

As in the formal case, if we are given an object $(E, \nabla)$ in $M I C\left(X_{K} / K\right)^{\log }$ and étale locally a cartesian diagram

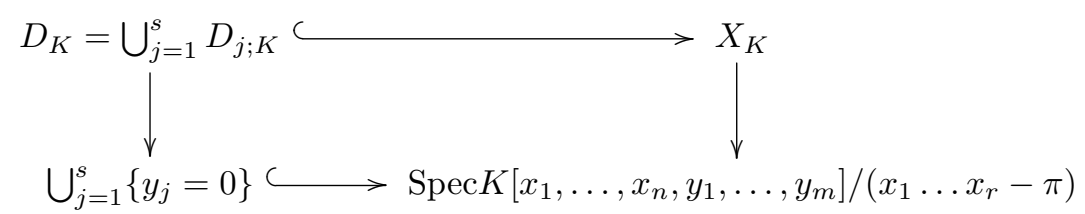

with the horizontal maps closed immersions and the vertical maps étale, we can define the exponents of $(E, \nabla)$ along each $D_{j ; K}:=D_{j} \otimes_{V} K$. Also, we can globalize the definition of exponents as in definition 3.11 in the following way.

Definition 4.7. We fix a set $\Sigma=\prod_{h=1}^{t} \Sigma_{h} \subset \bar{K}^{t}$, where $t$ is the number of irreducible components of the divisor $D_{K}=\cup_{h=1}^{t} D^{h ; K}$ (which is equal to the number of irreducible components of $D$ ). We say that an object $(E, \nabla)$ in $M I C\left(\left(X_{K}, M\right) / K\right)$ has exponents in $\Sigma$ if there exists an étale covering $\coprod_{l} \phi_{l ; K}: \coprod_{l} X_{l ; K} \rightarrow X_{K}$ such that every $X_{l ; K}$ has a diagram

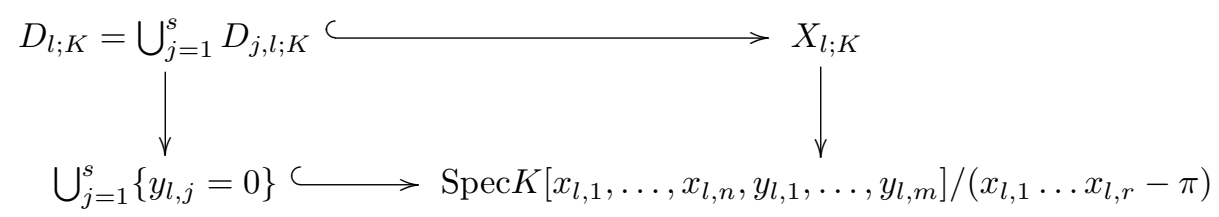

as in (4) with $D_{l ; K}:=\phi_{l ; K}^{-1}\left(D_{K}\right)$ and for for every $l$ and $j$, the restriction of $(E, \nabla)$ to $X_{l ; K}$ has exponents in $\Sigma_{h(j)}$ along $D_{j, l ; K}$, where $\phi_{l ; K}\left(D_{j, l ; K}\right) \subset D^{h(j) ; K}$. We denote by $M I C\left(X_{K} / K\right)^{\log , \Sigma}$ the category of modules with integrable connection on $\left(X_{K}, M\right)$ over $K$ with exponents in $\Sigma$.

Also in this case, one can prove that the above definition does not depend on the choice of the étale covering and the diagram (5).

In [7] II, 1] I.3 and 22] section 2, the notion of regularity along a normal crossing divisor which is union of smooth components is introduced. Since the irreducible components of $D_{K}$ are not necessarily smooth, we need a slight generalization of this notion.

Definition 4.8. We say that an object $(E, \nabla)$ in $M I C\left(U_{K} / K\right)$ is regular along $D_{K}$ if, around every generic point of $D_{K}$, there exists an étale neighborhood $X_{l ; K} \rightarrow X$ and a diagram as in (5) such that the module with integrable connection induced on $U_{l ; K}:=X_{l ; K} \backslash D_{l ; K}$ is regular along $D_{l ; K}$.

Proposition 4.9. The notion of regularity along $D_{K}$ is independent of the choice of étale neighborhood and the diagram chosen in definition 4.8 . 
Proof. It is essentially proved in [1] I. 3.4.4, 3.4.5. Indeed, let us take another etale neighborhood $X_{l ; K}^{\prime} \rightarrow X$ and a diagram as in (5) (which we denote by the same symbols with '). We want to prove that the pull-back of $(E, \nabla)$ to $U_{l ; K}^{\prime}:=X_{l ; K}^{\prime} \backslash D_{l ; K}^{\prime}$ is regular along $D_{l ; K}^{\prime}$. If we consider the fiber product $U_{l ; K} \times_{U_{K}} U_{l ; K}^{\prime}$, the pull-back of $(E, \nabla)$ to it is regular along the pull-back of $D_{K}$ by proposition I. 3.4.4 of [1], and it implies the regularity of the pull-back of $(E, \nabla)$ to $U_{l ; K}^{\prime}$ again by proposition I. 3.4.4 of [1].

There is a notion of exponents for an object of $\operatorname{MIC}\left(U_{K} / K\right)$ regular along $D_{K}$, which is defined in [1] I. 6.3. As before we need to adapt this notion to our situation.

Definition 4.10. Let $(E, \nabla)$ be an object in $M I C\left(U_{K} / K\right)$ regular along $D_{K}$. We fix a set $\bar{\Sigma}=\prod_{h=1}^{t} \bar{\Sigma}_{h} \subset$ $\bar{K}^{t} / \mathbb{Z}^{t}$, where $t$ is the number of irreducible components of the divisor $D_{K}=\cup_{h=1}^{t} D^{h ; K}$. We say that $E$ has exponents in $\bar{\Sigma}$ if, around every generic point of $D_{K}$, there exists an étale neighborhood $\phi_{l ; K}: X_{l ; K} \rightarrow X_{K}$ and a diagram (5) such that the module with integrable connection induced on $U_{l ; K}:=X_{l ; K} \backslash D_{l ; K}$ has exponents in the sense of [1] I. 6.3 along $D_{j, l ; K}$ contained in $\bar{\Sigma}_{h(j)}$ for every $j$, where $\phi_{l ; K}\left(D_{j, l ; K}\right) \subset D^{h(j) ; K}$. We denote the category of modules with integrable connection on $U_{K}$ regular along $D_{K}$ with exponents in $\bar{\Sigma}$ by $\operatorname{MIC}\left(U_{K} / K\right)^{r e g, \bar{\Sigma}}$.

Proposition 4.11. The notion of exponents in $\bar{\Sigma}$ for a module with integrable connection on $U_{K}$ regular along $D_{K}$ is independent of the choice of the étale neighborhood and the diagram chosen in definition 4.10.

Proof. We can proceed as in proposition 4.9, using proposition [1] I.6.4.3.

If we take a set $\bar{\Sigma}=\prod_{h=1}^{t} \bar{\Sigma}_{h} \subset \bar{K}^{t} / \mathbb{Z}^{t}$ and a section $\tau: \bar{K} / \mathbb{Z} \rightarrow \bar{K}$ of the canonical projection, the log extension theorem of André and Baldassarri ([1] I. 4.9) reads as follows.

Theorem 4.12. Given $\bar{\Sigma}=\prod_{h=1}^{t} \bar{\Sigma}_{h} \subset(\bar{K} / \mathbb{Z})^{t}$, where $t$ is the number of irreducible components of the divisor $D_{K}=\cup_{h=1}^{t} D^{h ; K}$. For every section $\tau: \bar{K} / \mathbb{Z} \rightarrow \bar{K}$, the restriction functor

$$
P_{\tau}: M I C\left(X_{K} / K\right)^{\log , \tau(\bar{\Sigma})} \longrightarrow M I C\left(U_{K} / K\right)^{r e g, \bar{\Sigma}},
$$

which is induced by the tensor functor

$$
P: M I C\left(X_{K} / K\right)^{\log } \longrightarrow M I C\left(U_{K} / K\right)^{r e g},
$$

is a well-defined functor which is an equivalence of categories, where $\tau(\bar{\Sigma}):=\prod_{h=1}^{t} \tau\left(\bar{\Sigma}_{h}\right)$.

Proof. We can work étale locally, apply [1] I.4.9, and use étale descent.

Proposition 4.13. If $\bar{\Sigma}$ is a subgroup of $\bar{K}^{t} / \mathbb{Z}^{t}$, the category $M I C\left(U_{K} / K\right)^{\text {reg }, \bar{\Sigma}}$ is a rigid abelian tensor category.

Proof. It follows from proposition I.6.4.2 of [1].

Finally in this section, we recall the results in [10] on the functors relating the categories $I_{\text {conv }}\left(X_{k} / V\right)^{\log }$, $\widehat{M I C}(\hat{X} / V)^{\log }$ and $M I C\left(X_{K} / K\right)^{\log }$.

Theorem 4.14. Let $\bar{\Sigma}=\prod_{h=1}^{t} \Sigma_{h}$ be a subset of $\left(\mathbb{Z}_{p} / \mathbb{Z}\right)^{t}$ and $\tau: \mathbb{Z}_{p} / \mathbb{Z} \rightarrow \mathbb{Z}_{p}$ be a section. Then there exists a fully faithful functor

$$
\Phi_{\tau}: I_{\text {conv }}\left(X_{k} / V\right)^{\log , \tau(\bar{\Sigma})} \longrightarrow \widehat{M I C}(\hat{X} / V)^{\log , \tau(\bar{\Sigma})}
$$

which is induced by the fully faithful tensor functor

$$
\Phi: I_{\text {conv }}\left(X_{k} / V\right)^{\log } \longrightarrow \widehat{M I C}(\hat{X} / V)^{\log }
$$

of 'forgetting the convergence condition'. Also, there exists an equivalence

$$
\Gamma_{\tau}: M I C\left(X_{K} / K\right)^{\log , \tau(\bar{\Sigma})} \longrightarrow \widehat{M I C}(\hat{X} / V)^{\log , \tau(\bar{\Sigma})}
$$

which is induced by the GAGA equivalence

$$
\Gamma: M I C\left(X_{K} / K\right)^{\log } \longrightarrow \widehat{M I C}(\hat{X} / V)^{\log } .
$$


Proof. The functor $\Phi$ is the composition of the fully faithful tensor functor $\tilde{\Phi}$ in section 5 of [10] and the equivalence in proposition 3 and 4 in section 5 in [10. The equivalence $\Gamma$ is the composition of the equivalence $\tilde{\Psi}$ in section 5 of [10] and an equivalence induced by theorem 3.2.15 of [12]. Also, it is easy to see that $\Phi, \Gamma$ induce the functors $\Phi_{\tau}, \Gamma_{\tau}$ respectively.

\section{Properties of algebraization functor}

The main result of [10 can be translated in the context of this paper as follows: if $\bar{\Sigma}$ is (NLG), then we constructed a fully faithful functor

$$
\psi_{\tau}: I^{\dagger}\left(U_{k} / V\right)^{\log , \bar{\Sigma}} \longrightarrow M I C\left(U_{K} / K\right)^{r e g, \bar{\Sigma}},
$$

which is the composition of the four functors

$$
\begin{aligned}
& j_{\tau}^{\dagger,-1}: I^{\dagger}\left(U_{k} / V\right)^{\log , \bar{\Sigma}} \longrightarrow I_{\text {conv }}\left(X_{k} / V\right)^{\log , \tau(\bar{\Sigma})}, \\
& \Phi_{\tau}: I_{\text {conv }}\left(X_{k} / V\right)^{\log , \tau(\bar{\Sigma})} \longrightarrow \widehat{M I C}(\hat{X} / V)^{\log , \tau(\bar{\Sigma})}, \\
& \Gamma_{\tau}^{-1}: \widehat{M I C}(\hat{X} / V)^{\log , \tau(\bar{\Sigma})} \longrightarrow M I C\left(X_{K} / K\right)^{\log , \tau(\bar{\Sigma})}, \\
& P_{\tau}: M I C\left(X_{K} / K\right)^{\log , \tau(\bar{\Sigma})} \longrightarrow M I C\left(U_{K} / K\right)^{r e g, \bar{\Sigma}},
\end{aligned}
$$

where the first one is the quasi-inverse of the functor $j_{\tau}^{\dagger}$ in theorem 3.13 the second one is as in theorem 4.14 the third one is the quasi-inverse of the functor $\Gamma_{\tau}$ in theorem 4.14 and the fourth one is the functor in theorem 4.12

We want to stress that there are tensor functors

$$
\begin{aligned}
& j^{\dagger}: I_{\mathrm{conv}}\left(X_{k} / V\right)^{\log } \longrightarrow I^{\dagger}\left(U_{k} / V\right)^{\log }, \\
& \Phi: I_{\mathrm{conv}}\left(X_{k} / V\right)^{\log } \longrightarrow \widehat{M I C}(\hat{X} / V)^{\log }, \\
& \Gamma^{-1}: \widehat{M I C}(\hat{X} / V)^{\log } \longrightarrow M I C\left(X_{K} / K\right)^{\log }, \\
& P: M I C\left(X_{K} / K\right)^{\log } \longrightarrow M I C\left(U_{K} / K\right)^{r e g},
\end{aligned}
$$

extending $j_{\tau}^{\dagger}, \Phi_{\tau}, \Gamma_{\tau}^{-1}, P_{\tau}$ respectively.

The first main theorem in this paper is the following.

Theorem 5.1. The functor $\psi_{\tau}$ is a tensor functor which is independent of the choice of $\tau$.

To prove the theorem, first we prove a certain property close to the full-faithfulness of the functor $j^{\dagger}$.

Proposition 5.2. Let $\Sigma_{i}:=\prod_{h=1}^{t} \Sigma_{i, h}(i=1,2)$ be subsets of $\mathbb{Z}_{p}^{t}$ which are (NLD) and (NID) and suppose that, for every $h$ and every $\xi_{i} \in \Sigma_{i, h}(i=1,2), \xi_{2}-\xi_{1}$ belongs to $\mathbb{Z}_{p} \backslash \mathbb{Z}_{>0}$. Let $\mathcal{E}_{i}$ be an object in $I_{\text {conv }}\left(X_{k} / V\right)^{\log , \Sigma_{i}}$ for $i=1,2$. Then the functor $j^{\dagger}$ induces the isomorphism

$$
\operatorname{Hom}\left(\mathcal{E}_{1}, \mathcal{E}_{2}\right) \cong \operatorname{Hom}\left(j^{\dagger} \mathcal{E}_{1}, j^{\dagger} \mathcal{E}_{2}\right) .
$$

Proof. The argument is analogous to the one in proposition 1.18 of [16].

Given a morphism $f: j^{\dagger} \mathcal{E}_{1} \rightarrow j^{\dagger} \mathcal{E}_{2}$ of log overconvergent isocrystals, we should prove that $f$ extends uniquely to a morphism $\tilde{f}: \mathcal{E}_{1} \rightarrow \mathcal{E}_{2}$. We can work étale locally with the diagram (2), using étale descent for log convergent isocrystals (remark 5.1.7 of [12]) and for log overconvergent isocrystals (lemma 4.3 of [13]). Let us take a small enough strict neighborhood $W$ of $] U_{k}[\hat{X}$ in $] X_{k}\left[\hat{X}_{\hat{X}}\right.$ so that there exists a morphism $\phi: E_{1}^{\prime} \rightarrow E_{2}^{\prime}$ of coherent modules with integrable connection on $W$ such that $j_{W}^{\dagger} \phi=f$, where $j_{W}^{\dagger}$ is the functor of associating the sheaf of overconvergent sections ((2.1.1.1) of [4). We denote by $E_{i}(i=1,2)$ the $\log -\nabla$-module induced by $\mathcal{E}_{i}$ on $\hat{X}_{K}$ (with respect to $y_{1}, \ldots, y_{s}$ ). It suffices to extend the morphism $\phi$ uniquely to a morphism $E_{1} \rightarrow E_{2}$. 
Let us take $\lambda \in(0,1) \cap \Gamma^{*}$ and the covering

$$
\hat{X}_{K}=\bigcup_{J \subset\{1 \ldots s\}} A_{J}
$$

of $\hat{X}_{K}$, where

$$
\begin{aligned}
A_{J} & =\left\{P \in \hat{X}_{K}|| y_{j}(P)|<1 \quad(j \in J)| y_{j}(P) \mid \geq \lambda(j \notin J)\right\} \\
& =\left\{P \in \hat{X}_{K}\left|y_{j}(P)=0(j \in J),\right| y_{j}(P) \mid \geq \lambda(j \notin J)\right\} \times A^{|J|}[0,1) .
\end{aligned}
$$

Also we suppose that we chose $\lambda$ such that $E_{i}^{\prime}$ 's are defined on the following set:

$$
B=\left\{P \in \hat{X}_{K}|| y_{j}(P) \mid \geq \lambda \forall j\right\}
$$

The covering of $\hat{X}_{K}$ given by the $A_{J}$ 's restricts to the covering $B=\bigcup_{J \subset\{1, \ldots, s\}} B_{J}$, where

$$
\begin{aligned}
B_{J} & =\left\{P \in \hat{X}_{K}|\lambda \leq| y_{j}(P)|<1(j \in J), \quad| y_{j}(P) \mid \geq \lambda(j \notin J)\right\} \\
& =\left\{P \in \hat{X}_{K}\left|y_{j}(P)=0(j \in J)\right| y_{j}(P) \mid \geq \lambda(j \notin J)\right\} \times A^{|J|}[\lambda, 1) .
\end{aligned}
$$

We can check that $E_{1}, E_{2}$ are log-convergent in the sense of [15] on

$$
A_{J}=\left\{P \in \hat{X}_{K}\left|y_{j}(P)=0(j \in J),\right| y_{j}(P) \mid \geq \lambda(j \notin J)\right\} \times A^{|J|}[0,1)
$$

in the same way as proposition 3.6 of [15] and they have exponents in $\prod_{j} \Sigma_{1, h(j)}, \prod_{j} \Sigma_{2, h(j)}$, respectively. So, by proposition 2.12 of [15], they are $\prod_{j} \Sigma_{1, h(j)}$-unipotent, $\prod_{j} \Sigma_{2, h(j)}$-unipotent respectively. Hence, by proposition 5.3 below, the canonical map

$$
\operatorname{Hom}_{A_{J}}\left(E_{1}, E_{2}\right) \rightarrow \operatorname{Hom}_{B_{J}}\left(E_{1}^{\prime}, E_{2}^{\prime}\right)
$$

is an isomorphism. So the restriction $\phi_{J}$ of $\phi$ to $B_{J}$ extends uniquely to a morphism $E_{1} \rightarrow E_{2}$ on $A_{J}$ for every $J$. On $A_{I} \cap A_{J}$ we have the extensions $\phi_{I}$ and $\phi_{J}$, which glue again by proposition 5.3 below because they coincide on the set

$$
\begin{aligned}
& B_{I} \cap B_{J}=\left\{P \in \hat{X}_{K}|\lambda \leq| y_{j}(P) \mid<1\right.(j \in(I \cup J)-(I \cap J)), \\
&\left.\left|y_{j}(P)\right| \geq \lambda(j \notin(I \cup J))\right\} \times A^{|I \cap J|}[\lambda, 1),
\end{aligned}
$$

both extending the restriction of $\phi$ on $B_{I} \cap B_{J}$. So we obtain the desired extension of $\phi$ to $\hat{X}_{K}$.

Proposition 5.3. Let $Y$ be a smooth rigid space over $K$, let $\lambda$ be an element in $(0,1) \cap \Gamma^{*}$ and let $\Sigma_{1}:=$ $\prod_{j=1}^{n} \Sigma_{1, j}, \Sigma_{2}:=\prod_{j=1}^{n} \Sigma_{2, j}$ be subsets of $\mathbb{Z}_{p}^{n}$. For $i=1,2$, let $E_{i}$ be a $\Sigma_{i}$-unipotent log- $\nabla$-module on $Y \times A_{K}^{n}[0,1)$ and let us put $E_{i}^{\prime}=E_{i \mid Y \times A_{K}^{n}[\lambda, 1)}$, which is a log- $\nabla$-module on $Y \times A_{K}^{n}[\lambda, 1)$. If for any $j$ and for any $\xi_{1} \in \Sigma_{1, j, E_{1}}, \xi_{2} \in \Sigma_{2, j, E_{2}}$ we have $\xi_{2}-\xi_{1} \in \mathbb{Z}_{p} \backslash \mathbb{Z}_{>0}$, the restriction functor induces an isomorphism of the following groups of homomorphisms of log- $\nabla$-modules

$$
\operatorname{Hom}\left(E_{1}, E_{2}\right) \cong \operatorname{Hom}\left(E_{1}^{\prime}, E_{2}^{\prime}\right) .
$$

Proof. This is proposition 1.6 of [16].

We prove the independence of the functor $\psi_{\tau}$ of the choice of $\tau$.

Proposition 5.4. The functor $\psi_{\tau}$ is independent of the choice of $\tau$. 
Proof. Let us take sections $\tau_{1}, \tau_{2}: \mathbb{Z}_{p} / \mathbb{Z} \rightarrow \mathbb{Z}_{p}$. Define $\tau_{3}: \mathbb{Z}_{p} / \mathbb{Z} \rightarrow \mathbb{Z}_{p}$ by

$$
\tau_{3}(\xi):= \begin{cases}\tau_{1}(\xi), & \text { if } \tau_{1}(\xi)-\tau_{2}(\xi) \in \mathbb{Z}_{\leq 0}, \\ \tau_{2}(\xi), & \text { if } \tau_{1}(\xi)-\tau_{2}(\xi) \in \mathbb{Z}_{>0} .\end{cases}
$$

Then, for any $\xi \in \mathbb{Z}_{p} / \mathbb{Z}, \tau_{3}(\xi)-\tau_{1}(\xi), \tau_{3}(\xi)-\tau_{2}(\xi)$ belong to $\mathbb{Z}_{p} \backslash \mathbb{Z}_{>0}$. Hence, by replacing $\tau_{1}$ or $\tau_{2}$ by $\tau_{3}$, we are reduced to prove the isomorphism $\psi_{\tau_{1}} \cong \psi_{\tau_{2}}$ in the case where $\tau_{2}(\xi)-\tau_{1}(\xi) \in \mathbb{Z}_{p} \backslash \mathbb{Z}_{>0}$ for any $\xi \in \mathbb{Z}_{p} / \mathbb{Z}$

Let us take an object $\mathcal{E}$ in $I^{\dagger}\left(U_{k} / V\right)^{\log , \bar{\Sigma}}$ and put $\mathcal{E}_{i}:=j_{\tau_{i}}^{\dagger,-1}(\mathcal{E})$. Then, by proposition 5.2 , we have the unique morphism $\mu: \mathcal{E}_{1} \rightarrow \mathcal{E}_{2}$ in $I_{\text {conv }}\left(X_{k} / V\right)^{\log }$ which extends the identity on $\mathcal{E}$. By applying $P \circ \Gamma^{-1} \circ \Phi$, we obtain the morphism $f: \psi_{\tau_{1}}(\mathcal{E}) \rightarrow \psi_{\tau_{2}}(\mathcal{E})$. It suffices to prove that $f$ is an isomorphism.

For an integer $\alpha$, we can define the $\log$ convergent isocrystal $\mathcal{O}\left(\alpha D_{k}\right)$ as in 16 proposition 3.1, in the following way: We denote by $\tau^{\alpha}: \mathbb{Z}_{p} / \mathbb{Z} \rightarrow \mathbb{Z}_{p}$ any section with $\tau^{\alpha}(0)=-\alpha$. By theorem 3.13 if $\mathbf{0}=(\overline{0}, \ldots, \overline{0})$, we know that the functor

$$
j_{\tau^{\alpha}}^{\dagger}: I_{\text {conv }}(\hat{X} / V)^{\log , \tau^{\alpha}(\mathbf{0})} \longrightarrow I^{\dagger}\left(U_{k} / V\right)^{\log , \mathbf{0}}
$$

is an equivalence of categories. Hence we have the unique object in $I_{\text {conv }}(\hat{X} / V)^{\log , \tau^{\alpha}(\mathbf{0})}$ which extends the trivial log overconvergent isocrystal in $I^{\dagger}\left(U_{k} / V\right)^{\log , \mathbf{0}}$. We denote it by $\mathcal{O}\left(\alpha D_{k}\right)$. By considering the description of it in terms of (formal) log connections as in [16] proposition 3.1, we see that $\Gamma^{-1} \circ \Phi$ sends $\mathcal{O}\left(\alpha D_{k}\right)$ to the object $\left(\mathcal{O}_{X_{K}}\left(\alpha D_{K}\right), d\right)$ in $M I C\left(X_{K} / K\right)^{\log }$, and so it is sent to the trivial object in $M I C\left(U_{K} / K\right)^{r e g}$ by the functor $P$. Also, when $\alpha \geq 0$, the unique morphism $\iota: \mathcal{O} \rightarrow \mathcal{O}\left(\alpha D_{k}\right)$ in $I_{\text {conv }}(\hat{X} / V)^{\log }$ (where $\mathcal{O}$ is the trivial object) extending the identity (whose existence follows from proposition 5.2 is sent to the identity map.

Now, let $\bar{\Sigma}_{\mathcal{E}}=\prod_{h} \bar{\Sigma}_{\mathcal{E}, h}$ be as in definition 3.14 and let $\alpha$ be an element in $\mathbb{Z}_{\geq 0}$ satisfying the following condition: for any $h$ and any $\xi_{i} \in \tau_{i}\left(\bar{\Sigma}_{\mathcal{E}, h}\right)(i=1,2), \xi_{1}-\xi_{2}-\alpha$ belongs to $\mathbb{Z}_{p} \backslash \mathbb{Z}_{>0}$. (Such $\alpha$ exists because $\bar{\Sigma}_{\mathcal{E}}$ is a finite set.) If we put $\mathcal{E}_{1}\left(\alpha D_{k}\right):=\mathcal{E}_{1} \otimes \mathcal{O}\left(\alpha D_{k}\right)$, it is an object in $I_{\text {conv }}(\hat{X} / V)^{\log , \tau_{1}\left(\bar{\Sigma}_{\mathcal{E}}\right)-\boldsymbol{\alpha}}$ (where $\boldsymbol{\alpha}=(\alpha, \ldots, \alpha))$ and $\mathcal{E}_{2}$ is an object in $I_{\text {conv }}(\hat{X} / V)^{\log , \tau_{2}\left(\bar{\Sigma}_{\mathcal{E}}\right)}$. Hence, by proposition 5.2 , we have the unique morphism $\mu^{\prime}: \mathcal{E}_{2} \rightarrow \mathcal{E}_{1}\left(\alpha D_{k}\right)$ extending the identity on $\mathcal{E}$, and by sending it by $P \circ \Gamma^{-1} \circ \Phi$, we obtain the morphism $g: \psi_{\tau_{2}}(\mathcal{E}) \rightarrow \psi_{\tau_{1}}(\mathcal{E}) \otimes\left(P \circ \Gamma^{-1} \circ \Psi\right)\left(\mathcal{O}\left(\alpha D_{k}\right)\right)=\psi_{\tau_{1}}(\mathcal{E})$. By the unicity of $\mu$ and $\mu^{\prime}$, the composite $\mu^{\prime} \circ \mu$ is equal to the map id $\mathcal{E}_{1} \otimes \iota$. Hence, by applying $P \circ \Gamma^{-1} \circ \Phi$, we see that the composite $g \circ f$ is equal to the identity map.

Now we repeat the same argument: we have the unique morphism $\mu^{\prime \prime}: \mathcal{E}_{2}\left(-\alpha D_{k}\right) \rightarrow \mathcal{E}_{1}$ extending the identity on $\mathcal{E}$, which is by unicity equal to $\mu^{\prime} \otimes \operatorname{id}_{\mathcal{O}\left(-\alpha D_{k}\right)}$. By applying $P \circ \Gamma^{-1} \circ \Phi$, we obtain the morphism $g: \psi_{\tau_{2}}(\mathcal{E}) \rightarrow \psi_{\tau_{1}}(\mathcal{E})$ same as before. By the argument in the previous paragraph, we see that the composite $f \circ g$ is also equal to the identity map. Hence we see that $f$ is an isomorphism, and so we are done.

Next we prove that the functor $\psi_{\tau}$ is a tensor functor.

Proposition 5.5. The functor $\psi_{\tau}$ is a tensor functor.

Proof. Let $\mathcal{E}_{i}(i=1,2)$ be objects in $I^{\dagger}\left(U_{k} / V\right)^{\log , \bar{\Sigma}}$ and let $\bar{\Sigma}_{\mathcal{E}_{i}}:=\prod_{h} \bar{\Sigma}_{\mathcal{E}_{i}, h}(i=1,2), \bar{\Sigma}_{\mathcal{E}_{1} \otimes \mathcal{E}_{2}}:=\prod_{h} \bar{\Sigma}_{\mathcal{E}_{1} \otimes \mathcal{E}_{2}, h}$ be as in definition 3.14 Let us take a section $\tau^{\prime}: \mathbb{Z}_{p} / \mathbb{Z} \rightarrow \mathbb{Z}_{p}$ satisfying the following condition: For any $h$ and for any $a_{i} \in \bar{\Sigma}_{\mathcal{E}_{i}, h}(i=1,2), b \in \bar{\Sigma}_{\mathcal{E}_{1} \otimes \mathcal{E}_{2}, h}, \tau\left(a_{1}\right)+\tau\left(a_{2}\right)-\tau^{\prime}(b) \in \mathbb{Z}_{p} \backslash \mathbb{Z}_{>0}$. (It is possible to take such $\tau^{\prime}$ because $\bar{\Sigma}_{\mathcal{E}_{i}}, \bar{\Sigma}_{\mathcal{E}_{1} \otimes \mathcal{E}_{2}}$ are finite sets.) Put $\mathcal{F}_{1}:=j_{\tau^{\prime}}^{\dagger,-1}\left(\mathcal{E}_{1} \otimes \mathcal{E}_{2}\right), \mathcal{F}_{2}:=j_{\tau}^{\dagger,-1}\left(\mathcal{E}_{1}\right) \otimes j_{\tau}^{\dagger,-1}\left(\mathcal{E}_{2}\right)$. Then, $\mathcal{F}_{1}$ belongs to $I_{\text {conv }}\left(X_{k} / V\right)^{\log , \tau^{\prime}\left(\bar{\Sigma}_{\mathcal{E}_{1} \otimes \mathcal{E}_{2}}\right)}$ and $\mathcal{F}_{2}$ belongs to $I_{\text {conv }}\left(X_{k} / V\right)^{\log , \tau\left(\bar{\Sigma}_{\mathcal{E}_{1}}\right)+\tau\left(\bar{\Sigma}_{\mathcal{E}_{2}}\right)}$. Hence, by definition of $\tau^{\prime}$ and proposition 5.2 , there exists the unique morphism $\mu: \mathcal{F}_{1} \rightarrow \mathcal{F}_{2}$ extending the identity morphism on $\mathcal{E}_{1} \otimes \mathcal{E}_{2}$. By applying $P \circ \Gamma^{-1} \circ \Phi$, we obtain the morphism $f: \psi_{\tau^{\prime}}\left(\mathcal{E}_{1} \otimes \mathcal{E}_{2}\right) \rightarrow \psi_{\tau}\left(\mathcal{E}_{1}\right) \otimes \psi_{\tau}\left(\mathcal{E}_{2}\right)$.

Then, by the same argument as the proof of the previous proposition (using $\mathcal{O}\left(\alpha D_{k}\right)$ for some $\alpha$ ), we see that the morphism $f$ is an isomorphism. By combining this with the isomorphism $\psi_{\tau^{\prime}}\left(\mathcal{E}_{1} \otimes \mathcal{E}_{2}\right) \cong \psi_{\tau}\left(\mathcal{E}_{1} \otimes \mathcal{E}_{2}\right)$ of the previous proposition, we obtain the isomorphism

$$
\psi_{\tau}\left(\mathcal{E}_{1} \otimes \mathcal{E}_{2}\right) \cong \psi_{\tau}\left(\mathcal{E}_{1}\right) \otimes \psi_{\tau}\left(\mathcal{E}_{2}\right),
$$

as desired. 
By propositions 5.4 and 5.5 , the proof of theorem 5.1 is finished.

Next we prove the following theorem, which is the second main result in this paper.

Theorem 5.6. The essential image of the functor $\psi_{\tau}$ is closed under extensions and subquotients.

In order to prove this theorem, it suffices to prove the same property for the functor

$$
\Phi_{\tau}: I_{\text {conv }}\left(X_{k} / V\right)^{\log , \tau(\bar{\Sigma})} \longrightarrow \widehat{M I C}(\hat{X} / V)^{\log , \tau(\bar{\Sigma})},
$$

because the functors $j_{\tau}^{\dagger,-1}, \Gamma_{\tau}^{-1}, P_{\tau}$ are equivalences.

Also, it is easy to see that the condition on exponent is closed under extensions and subquotients. Hence it suffices to prove the same property for the functor

$$
\Phi: I_{\text {conv }}\left(X_{k} / V\right)^{\log } \longrightarrow \widehat{M I C}(\hat{X} / V)^{\log } .
$$

Then, by proposition 8 of [10, one can replace $\hat{X}$ by $\hat{U}$ to prove it.

Now we recall the definition of formal stratifications and special formal stratifications (definition 3.2.10 of [12], definition 15 of [10]).

Definition 5.7. We define the category $\widehat{\operatorname{Str}}(\hat{U} / V)$ of formal stratifications on $\hat{U}$ as follows: An object is a pair $\left(E,\left\{\epsilon_{n}\right\}\right)$ of a locally free isocoherent sheaf $E$ on $\hat{U}$ and a compatible family $\left\{\epsilon_{n}: \mathcal{O}_{\hat{U}^{n}} \otimes E \rightarrow E \otimes \mathcal{O}_{\hat{U}^{n}}\right\}_{n \in \mathbb{N}}$ of linear isomorphisms (where $\hat{U}^{n}$ is the $n$-th infinitesimal neighborhood of $\hat{U}$ in $\hat{U} \times{ }_{V} \hat{U}$ ) satisfying the cocycle condition on the infinitesimal neighborhoods of $\hat{U}$ in $\hat{U} \times_{V} \hat{U} \times_{V} \hat{U}$.

Definition 5.8. Let $\left(E,\left\{\epsilon_{n}\right\}\right)$ be an object of $\widehat{\operatorname{Str}}(\hat{U} / V)$ and let $\tilde{E}$ be a coherent p-torsion-free $\mathcal{O}_{\hat{U}}$-module such that $K \otimes \tilde{E}=E$; we say that $\left(E,\left\{\epsilon_{n}\right\}\right)$ is special if there exists a sequence of integers $k(n)$ for $n \in \mathbb{N}$ such that:

(i) $k(n)=o(n)$ for $n \rightarrow \infty$,

(ii) the restriction of the map $p^{k(n)} \epsilon_{n}$ to $\mathcal{O}_{\hat{U}^{n}} \otimes \tilde{E}$ has image contained in $\tilde{E} \otimes \mathcal{O}_{\hat{U}^{n}}$ and the restriction of the map $p^{k(n)} \epsilon_{n}^{-1}$ to $\tilde{E} \otimes \mathcal{O}_{\hat{U}^{n}}$ has image contained in $\mathcal{O}_{\hat{U}^{n}} \otimes \tilde{E}$.

It is known that the definition of being special is independent of the choice of the coherent $p$-torsion-free $\mathcal{O}_{\hat{U}}$-module $\tilde{E}$. Hence the property of being special is a local property.

We proved in theorem 3.2.15 of [12] and in section 6 of [10] the following theorem, which characterizes the essential image of the functor $\Phi$ on $\hat{U}$.

Theorem 5.9. We have an equivalence of categories

$$
\widehat{M I C}(\hat{U} / V) \cong \widehat{S t r}(\hat{U} / V),
$$

and an object in $\widehat{\operatorname{Str}}(\hat{U} / V)$ belongs to the essential image of $\Phi: I_{\text {conv }}\left(U_{k} / V\right) \longrightarrow \widehat{M I C}(\hat{U} / V) \cong \widehat{S t r}(\hat{U} / V)$ if and only if it is special.

When $x_{1}, \ldots, x_{l}$ are local coordinates of $\hat{U}$ and $\partial_{1}, \ldots, \partial_{l}$ denote the corresponding derivations, the image of $(E, \nabla)$ by $[9]$ is given by $\left(E,\left\{\epsilon_{n}\right\}\right)$ with

$$
\epsilon_{n}=\sum_{|\boldsymbol{\beta}| \leq n} \frac{1}{\boldsymbol{\beta} !} \nabla_{\boldsymbol{\beta}} \cdot\left(1 \otimes x_{i}-x_{i} \otimes 1\right)^{\boldsymbol{\beta}}, \quad \nabla_{\boldsymbol{\beta}}:=\left(i d \otimes \partial_{1} \circ \nabla\right)^{\beta_{1}} \circ \cdots \circ\left(i d \otimes \partial_{l} \circ \nabla\right)^{\beta_{l}} .
$$

Note that $\epsilon_{n}^{-1}$ is given by

$$
\epsilon_{n}^{-1}=\sum_{|\boldsymbol{\beta}| \leq n} \frac{(-1)^{|\boldsymbol{\beta}|}}{\boldsymbol{\beta} !} \nabla_{\boldsymbol{\beta}} \cdot\left(1 \otimes x_{i}-x_{i} \otimes 1\right)^{\boldsymbol{\beta}}
$$

in the above situation.

By the above theorem, the proof of theorem 5.6 is reduced to the claim that the category of special formal stratifications is closed under taking extensions and subquotients, which we prove in the following two lemmata. 
Lemma 5.10. Let $\left(E,\left\{e_{n}\right\}\right),\left(F,\left\{f_{n}\right\}\right),\left(G,\left\{g_{n}\right\}\right)$ be objects in $\widehat{\operatorname{Str}}(\hat{U} / V)$ with $\left(E,\left\{e_{n}\right\}\right)$ and $\left(F,\left\{f_{n}\right\}\right)$ special and suppose that there exists an exact sequence

$$
0 \longrightarrow\left(E,\left\{e_{n}\right\}\right) \longrightarrow\left(G,\left\{g_{n}\right\}\right) \longrightarrow\left(F,\left\{f_{n}\right\}\right) \longrightarrow 0
$$

in $\widehat{\operatorname{Str}}(\hat{U} / V)$. Then $\left(G,\left\{g_{n}\right\}\right)$ is also special.

Proof. We can restrict to the local situation because being special is a local property. So we can take local coordinates $x_{1}, \ldots, x_{l}$ of $\hat{U}$ and the corresponding derivations $\partial_{1}, \ldots, \partial_{l}$. Also, we can suppose that $F$ is a projective $K \otimes \mathcal{O}_{\hat{X}}$-module. Then the exact sequence 110 splits as a sequence of isocoherent sheaves. We fix a splitting. Then we have an equality $G=E \oplus F$ as $K \otimes \mathcal{O}_{\hat{X}}$-modules. Also, we take $p$-torsion free coherent $\mathcal{O}_{\hat{X}}$-modules $\tilde{E}$ and $\tilde{F}$ with $K \otimes \tilde{E}=E$ and $K \otimes \tilde{F}=F$. Then we have the isomorphisms $K \otimes(\tilde{E} \oplus \tilde{F})=E \oplus F=G$.

Let us denote by $\left(E, \nabla_{E}\right),\left(F, \nabla_{F}\right)$ and $\left(G, \nabla_{G}\right)$ the modules with integrable connections associated to $\left(E,\left\{e_{n}\right\}\right),\left(F,\left\{f_{n}\right\}\right)$ and $\left(G,\left\{g_{n}\right\}\right)$ respectively. Then, by the exact sequence 10 and the splitting in the previous paragraph, we can write $\nabla_{G}\left(\partial_{i}\right)$ as the matrix

$$
\nabla_{G}\left(\partial_{i}\right)=\left(\begin{array}{cc}
\nabla_{E}\left(\partial_{i}\right) & B_{i} \\
0 & \nabla_{F}\left(\partial_{i}\right)
\end{array}\right)
$$

for some $B_{i}$. If we denote by $\nabla_{E, \boldsymbol{\beta}}, \nabla_{F, \boldsymbol{\beta}}, \nabla_{G, \boldsymbol{\beta}}$ the operator $\nabla_{\boldsymbol{\beta}}$ in theorem 9 for $\left(E, \nabla_{E}\right),\left(F, \nabla_{F}\right),\left(G, \nabla_{G}\right)$ respectively, we can calculate $\nabla_{G, \boldsymbol{\beta}}$ as

$$
\begin{aligned}
\frac{1}{\boldsymbol{\beta} !} \nabla_{G, \boldsymbol{\beta}}=\frac{1}{\boldsymbol{\beta} !} & \left(\begin{array}{cc}
\nabla_{E, \boldsymbol{\beta}} & 0 \\
0 & \nabla_{F, \boldsymbol{\beta}}
\end{array}\right) \\
& +\sum_{i=1}^{l} \sum_{b+c=\boldsymbol{\beta}-e_{i}} \frac{b ! c !}{\boldsymbol{\beta} !} \frac{1}{b !}\left(\begin{array}{cc}
\nabla_{E, b} & 0 \\
0 & \nabla_{F, b}
\end{array}\right)\left(\begin{array}{cc}
0 & B_{i} \\
0 & 0
\end{array}\right) \frac{1}{c !}\left(\begin{array}{cc}
\nabla_{E, c} & 0 \\
0 & \nabla_{F, c}
\end{array}\right),
\end{aligned}
$$

with $e_{i}=(0, \ldots, 1, \ldots, 0)$ with 1 at the $i$-th place. We know that there exists a sequence $l(n)$ of integers with $l(n)=o(n)$ as $n \rightarrow \infty$ such that

$$
p^{l(n)} \frac{1}{\boldsymbol{\beta} !} \nabla_{E, \boldsymbol{\beta}}(\tilde{E}) \subset \tilde{E}, \quad p^{l(n)} \frac{1}{\boldsymbol{\beta} !} \nabla_{F, \boldsymbol{\beta}}(\tilde{F}) \subset \tilde{F}
$$

for every $\boldsymbol{\beta}$ such that $|\boldsymbol{\beta}| \leq n$, because both $\left(E,\left\{e_{n}\right\}\right)$ and $\left(F,\left\{f_{n}\right\}\right)$ are special. If $\alpha$ is a positive integer such that $p^{\alpha} B_{i}(\tilde{E}) \subset \tilde{F}$ for $e \in \tilde{E}$ for any $i=1, \ldots, l$, then

$$
k(n)=l(n)+v_{p}\left(\frac{\boldsymbol{\beta} !}{b ! c !}\right)+\alpha
$$

is a sequence of integers such that

$$
p^{k(n)} \frac{1}{\boldsymbol{\beta} !} \nabla_{G, \boldsymbol{\beta}}(\tilde{E} \oplus \tilde{F}) \subset \tilde{E} \oplus \tilde{F}
$$

for every $\boldsymbol{\beta}$ such that $|\boldsymbol{\beta}| \leq n$. We know that $l(n)=o(n)$ for $n \rightarrow \infty$ and $\alpha$ does not depend on $n$, so that the only thing that we have to show is that

$$
\max _{|\boldsymbol{\beta}| \leq n, b+c=\boldsymbol{\beta}-e_{i}} v_{p}\left(\frac{\boldsymbol{\beta} !}{b ! c !}\right)=o(n),
$$

which is standard (see 12] proposition 5.2.14 for example).

In the next lemma we show that the category of special objects is closed by taking subquotients. 
Lemma 5.11. Let us suppose that we have an exact sequence in $\widehat{\operatorname{Str}}(\hat{U} / V)$ :

$$
0 \longrightarrow\left(E,\left\{e_{n}\right\}\right) \longrightarrow\left(G,\left\{g_{n}\right\}\right) \longrightarrow\left(F,\left\{f_{n}\right\}\right) \longrightarrow 0 .
$$

Then, if $\left(G,\left\{g_{n}\right\}\right)$ is special, then $\left(E,\left\{e_{n}\right\}\right)$ and $\left(F,\left\{f_{n}\right\}\right)$ are special.

Proof. As in lemma 5.10 we have the isomorphisms $K \otimes(\tilde{E} \oplus \tilde{F})=E \oplus F=G$ as $K \otimes_{V} \mathcal{O}_{\hat{U}}$-modules. By hypothesis, there exists a sequence of integers $k(n)$ with $k(n)=o(n)$ as $n \rightarrow \infty$ such that

$$
p^{k(n)} \frac{1}{\boldsymbol{\beta} !} \nabla_{G, \boldsymbol{\beta}}(\tilde{E} \oplus \tilde{F}) \subset \tilde{E} \oplus \tilde{F}
$$

for $|\boldsymbol{\beta}| \leq n$. By restricting $(12)$ to $E$, we obtain the inclusion

$$
p^{k(n)} \frac{1}{\boldsymbol{\beta} !} \nabla_{E, \boldsymbol{\beta}}(\tilde{E}) \subset \tilde{E}
$$

and this implies that $\left(E,\left\{e_{n}\right\}\right)$ is special. Also, by sending $(12)$ to $F$, we obtain the inclusion

$$
p^{k(n)} \frac{1}{\boldsymbol{\beta} !} \nabla_{F, \boldsymbol{\beta}}(\tilde{F}) \subset \tilde{F}
$$

and this implies that $\left(F,\left\{f_{n}\right\}\right)$ is also special.

Since the proof of lemmata 5.10, 5.11 are finished, the proof of theorem 5.6 is also finished.

We have the following corollary as an immediate consequence of theorems 5.1, 5.6.

Corollary 5.12. Let $\bar{\Sigma}=\prod_{h=1}^{t} \bar{\Sigma}_{h} \subset\left(\mathbb{Z}_{p} / \mathbb{Z}\right)^{t}$ be a subset which satisfies (NLG) and let us fix a section $\tau: \mathbb{Z}_{p} / \mathbb{Z} \rightarrow \mathbb{Z}_{p}$. Let $\mathcal{E}$ be an object in $I^{\dagger}\left(U_{k} / V\right)^{\log , \bar{\Sigma}}$. Then the functor $\psi_{\tau}$ induces an equivalence of rigid abelian tensor categories

$$
I^{\dagger}\left(U_{k} / V\right)^{\log , \bar{\Sigma}}(\mathcal{E}) \longrightarrow M I C\left(U_{K} / K\right)^{r e g, \bar{\Sigma}}\left(\psi_{\tau}(\mathcal{E})\right),
$$

where the left hand side (resp. the right hand side) is the smallest full subcategory containing $\mathcal{E}$ (resp. $\psi_{\tau}(\mathcal{E})$ ) and closed under extension, subquotient, tensor and dual.

Under a certain hypothesis, we have the following result which is a Tannakian interpretation of theorems 5.1, 5.6 and corollary 5.12 .

Theorem 5.13. Let $\bar{\Sigma}=\prod_{h=1}^{t} \bar{\Sigma}_{h} \subset\left(\mathbb{Z}_{p} / \mathbb{Z}\right)^{t}$ be a subset which satisfies (NLG) and let us fix a section $\tau: \mathbb{Z}_{p} / \mathbb{Z} \rightarrow \mathbb{Z}_{p}$. If there exists a $K$-rational point $x$ of $U_{K}$ and $U_{K}$ is connected, then the functor

$$
\psi_{\tau}: I^{\dagger}\left(U_{k} / V\right)^{\log , \bar{\Sigma}} \longrightarrow M I C\left(U_{K} / K\right)^{r e g, \bar{\Sigma}}
$$

is a functor of neutral Tannakian categories inducing the surjection of Tannaka duals. Also, for an object $\mathcal{E}$ in $I^{\dagger}\left(U_{k} / V\right)^{\log , \bar{\Sigma}}$, the functor

$$
\psi_{\tau}: I^{\dagger}\left(U_{k} / V\right)^{\log , \bar{\Sigma}}(\mathcal{E}) \longrightarrow M I C\left(U_{K}\right)^{r e g, \bar{\Sigma}}\left(\psi_{\tau}(\mathcal{E})\right)
$$

is an equivalence of neutral Tannakian categories (thus induces the isomorphism of Tannaka duals).

Proof. Thanks to theorems 5.1, 5.6, corollary 5.12 and proposition 2.21 in [9, it is enough to show the existence of a fiber functor from $I^{\dagger}\left(U_{k} / V\right)^{\log , \bar{\Sigma}}, M I C\left(U_{K} / K\right)^{r e g, \bar{\Sigma}}$ to the category of finite dimensional $K$-vector spaces. Let $x$ be the $K$-rational point that exists by hypothesis. We define a functor $\omega: M I C\left(U_{K} / K\right)^{r e g, \bar{\Sigma}} \rightarrow$ $V e c_{K}$ by $\left.(\mathcal{F}, \nabla) \mapsto \mathcal{F}\right|_{x}$, which is an exact tensor functor because $\mathcal{F}$ is locally free. To prove that is fully faithful we can use corollary 2.10 of $\left[\underline{8}\right.$, because $\operatorname{End}\left(\mathcal{O}_{U_{K}}, d\right)=K$ (which is proven for example in claim 1 of proposition 3.16 of [12]). Also, if we define the functor $\omega^{\prime}: I^{\dagger}\left(U_{k} / V\right)^{\log , \bar{\Sigma}} \rightarrow V e c_{K}$ by $\omega^{\prime}:=\omega \circ \psi_{\tau}$, it is also a fiber functor because $\operatorname{End}(\mathcal{O})=K$ also in the category $I^{\dagger}\left(U_{k} / V\right)^{\log , \bar{\Sigma}}$ because of the full faithfulness on $\psi_{\tau}$. 


\section{Acknowledgements}

The main part of this work was done when the first author was at the Graduate School of Mathematical Sciences of the University of Tokyo supported by a postdoctoral fellowship and kaken-hi (grant-in-aid) of the Japanese Society for the Promotion of Science (JSPS). She is now supported by a postdoctoral fellowship of Labex IRMIA. When the main part of this work was done, the second author was supported by JSPS Grantin-Aid for Young Scientists (B) 21740003 and Grant-in-Aid for Scientific Research (B) 22340001. Currently he is supported by JSPS Grant-in-Aid for Scientific Research (C) 25400008 and Grant-in-Aid for Scientific Research (B) 23340001.

\section{References}

[1] Y. André and F. Baldassarri, De Rham cohomology of differential modules on algebraic varieties, Progress in Mathematics 189, Birkhäuser, Basel, 2001, viii+214pp.

[2] F. Baldassarri, Towards an algebraic proof of Deligne's regularity criterion. an informal survey of open problems, Milan J. Math. 73 (2005), 237-258.

[3] F. Baldassarri and B. Chiarellotto, Formal and p-adic theory of differential systems with logarithmic singularities depending upon parameters, Duke Math. J. 72 (1993), no. 1, 241-300.

[4] P. Berthelot, Cohomologie rigide et cohomologie rigide à support propre. Première partie, prépublication de l'IRMAR 96-03 (1996).

[5] B. Chiarellotto and B. Le Stum, F-isocristaux unipotents, Compositio Math. 116 (1999), no. 1, $81-110$.

[6] _ Pentes en cohomologie rigide et F-isocristaux unipotents, Manuscripta Math. 100 (1999), no. 4, 455-468.

[7] P. Deligne, Equations différentielles à points singuliers réguliers, Lecture Notes in Mathematics 163, Springer-Verlag, Berlin-New York, 1970, iii+133pp.

[8] _ Catégories tannakiennes, The Grothendieck Festschrift, Vol. II, Progress in Mathematics 87, Birkhäuser Boston, Boston, MA, 1990, pp. 111-195.

[9] P. Deligne and J. S. Milne, Tannakian categories, Hodge cycles, motives, and Shimura varieties (Pierre Deligne, James S. Milne, Arthur Ogus, and Kuang-yen Shih, eds.), Lecture Notes in Mathematics 900, Springer-Verlag, Berlin, 1982, pp. 101-228.

[10] V. Di Proietto, On p-adic differential equations on semistable varieties, Math. Z. 274 (2013), 1047-1091.

[11] K. S. Kedlaya, Semistable reduction for overconvergent F-isocrystals. I. Unipotence and logarithmic extensions, Compositio Math. 143 (2007), no. 5, 1164-1212.

[12] A. Shiho, Crystalline fundamental groups. I. Isocrystals on log crystalline site and log convergent site, J. Math. Sci. Univ. Tokyo 7 (2000), no. 4, 509-656.

[13] _ Relative log convergent cohomology and relative rigid cohomology II, arXiv:0707.1743.

[14] _ Cut-by-curves criterion for the overconvergence of p-adic differential equations, Manuscripta Math. 132 (2010), no. 3-4, 517-537.

[15] _ On logarithmic extension of overconvergent isocrystals, Math. Ann. 348 (2010), 467-512.

[16] _ Parabolic log convergent isocrystals, arXiv:1010.4364. 
$[17] \_$, Cut-by-curves criterion for the log extendability of overconvergent isocrystals, Math. Z. 269 (2011), no. 1-2, 59-82. 